ARTICLE

https://doi.org/10.1038/s41467-019-13270-7

\title{
Interface-induced magnetic polar metal phase in complex oxides
}

Meng Meng ${ }^{1}$, Zhen Wang 1,2, Aafreen Fathima ${ }^{3}$, Saurabh Ghosh ${ }^{3,4,5 \star}$, Mohammad Saghayezhian (D) 1, Joel Taylor', Rongying Jin', Yimei Zhu², Sokrates T. Pantelides (1) 4,5, Jiandi Zhang ${ }^{1}$, E.W. Plummer ${ }^{1 \star}$ \& Hangwen Guo ${ }^{1 \star}$

Polar metals are commonly defined as metals with polar structural distortions. Strict symmetry restrictions make them an extremely rare breed as the structural constraints favor insulating over metallic phase. Moreover, no polar metals are known to be magnetic. Here we report on the realization of a magnetic polar metal phase in a $\mathrm{BaTiO}_{3} / \mathrm{SrRuO}_{3} / \mathrm{BaTiO}_{3}$ heterostructure. Electron microscopy reveals polar lattice distortions in three-unit-cells thick $\mathrm{SrRuO}_{3}$ between $\mathrm{BaTiO}_{3}$ layers. Electrical transport and magnetization measurements reveal that this heterostructure possesses a metallic phase with high conductivity and ferromagnetic ordering with high saturation moment. The high conductivity in the $\mathrm{SrRuO}_{3}$ layer can be attributed to the effect of electrostatic carrier accumulation induced by the $\mathrm{BaTiO}_{3}$ layers. Density-functional-theory calculations provide insights into the origin of the observed properties of the thin $\mathrm{SrRuO}_{3}$ film. The present results pave a way to design materials with desired functionalities at oxide interfaces.

\footnotetext{
${ }^{1}$ Department of Physics \& Astronomy, Louisiana State University, Baton Rouge, LA 70803, USA. ${ }^{2}$ Condensed Matter Physics \& Materials Science Department, Brookhaven National Laboratory, Upton, NY 11973, USA. ${ }^{3}$ Department of Physics \& Nanotechnology and SRM Research Institute, SRM Institute of Science and Technology, Kattankulathur 603203 Tamil Nadu, India. ${ }^{4}$ Department of Physics and Astronomy and Department of Electrical Engineering and Computer Science, Vanderbilt University, Nashville, Tennessee 37235, USA. ${ }^{5}$ Materials Science and Technology Division, Oak Ridge National Laboratory, Oak Ridge, Tennessee 37831, USA. `email: saurabhghosh2802@gmail.com; wplummer@phys.lsu.edu; hangwenguo@fudan.edu.cn
} 
$\mathrm{n}$ condensed matter physics, the itinerant electrons in metals lead to effective screening of local electric dipole moments, disfavoring the formation of long-range ordered dipoles, i.e. polar structural distortions ${ }^{1-3}$. Consequently, polar metals are rare in nature ${ }^{3-6}$. Inspired by the original work of Anderson and Blount ${ }^{4}$, an extensive search of polar metals has been conducted, especially in transition-metal compounds. In transition-metal oxides (TMOs), polar distortions exist in ferroelectric materials, which are typically insulating with empty $\mathrm{d}$ orbitals (e.g., $\mathrm{d}^{0}$ configuration $)^{7}$. Metallicity can be induced via carrier doping by creating oxygen vacancies or replacing transition-metal ions. However, the amplitudes of polar distortions are suppressed rapidly, as has been shown in the cases of $\mathrm{BaTiO}_{3-\delta}$ and $\mathrm{PbTi}_{1-x} \mathrm{Nb}_{x} \mathrm{O}_{3}{ }^{8-10}$. An alternative approach to achieve a stable polar metal phase is to induce polar distortions and metallicity from different types of ions. For example, in $\mathrm{LiOsO}_{3}$ bulk crystals ${ }^{11}$, polar distortions are induced at the A-site Li ions while metallicity is maintained with the partially filled B-site Os orbitals ${ }^{1,2}$. Similarly in $\mathrm{NdNiO}_{3}(111)$ thin films, Nd off-centering and partially filled $\mathrm{Ni}$ orbitals are responsible for polar distortions and conductivity, respectively ${ }^{12}$. In both cases, the coupling strength between polar distortions and itinerant electrons is intrinsically weak as the two features originate from different ion sublattices ${ }^{12}$. However, $\mathrm{LiOsO}_{3}$ is highly toxic and unsuited for thin-film fabrication due to the high Li ionic mobility, while the conductivity of $\mathrm{NdNiO}_{3}$ (111) thin films is low. Notably, no polar metals in the literature possess any pronounced magnetism. This fact fits in the scenario that magnetism usually requires exchange interactions between partially filled $\mathrm{d}$ states $\left(\mathrm{d}^{n}\right.$ configuration) where polar distortions are inhibited ${ }^{13}$.

In this article, we present an approach to establish a ferromagnetic polar metal phase using oxide heterostructures. Due to the strong interplay among spin, lattice, and charge degrees of freedom at the heterointerface ${ }^{14-18}$, in $\mathrm{BaTiO}_{3} / \mathrm{SrRuO}_{3} / \mathrm{BaTiO}_{3}$ (BTO/SRO/BTO) heterostructures, we demonstrate that polar distortions can be induced in 3-u.c. of SRO by interfacial effects as revealed by aberration corrected electron microscopy and spectroscopy. This 3-u.c. SRO exhibits high conductivity and ferromagnetism with high magnetic moment, thus forming a unique magnetic polar metal phase. Density-functional-theory (DFT) calculations provide insights into the origin of the observed properties of the thin SRO film. The polar distortions, metallicity, and magnetism in our system all originate from the $\mathrm{Ru}-\mathrm{O}$ atomic layers so that intrinsic coupling among these features is expected. The present results not only can lead to ferroelectric-like functionality in a metallic system ${ }^{19}$, but also indicate a pathway to design materials with emergent functionalities ${ }^{20,21}$.

\section{Results}

STEM structural characterization. A heterostructure consisting of 9 u.c. BTO/ 3 u.c. SRO/ 10 u.c. BTO (referred to as BTO 9/3/10 hereafter) is grown on a $\mathrm{SrTiO}_{3}$ (STO) substrate by pulsed laser deposition (see Methods). We first address lattice distortions in the heterostructure. Cross-sectional aberration corrected scanning transmission electron microscopy (STEM) combined with electron energy loss spectroscopy (EELS) serves as one of the most powerful tools to identify lattice distortions in ultrathin films across interfaces 22,23 . Figure 1a displays a high-angle annular dark field (HAADF)-STEM image of the BTO 9/3/10 film grown on a $\mathrm{TiO}_{2}$-terminated $\mathrm{STO}$ substrate. The image intensity is roughly proportional to the total atomic number $Z^{2}$ in the column. The SRO block starts and terminates with a SrO layer in agreement with the well-known SRO growth mode ${ }^{24}$. Detailed EELS analysis illustrates that a small amount of chemical intermixing is mostly confined within one unit cell at both sides of the interfaces (Supplementary Note 1 and Supplementary Fig. 1).
Bulk BTO exhibits a polar structure where titanium and oxygen ions displace against one another, whereas bulk SRO has a nonpolar antiferrodistrotive (AFD) structure with tilted oxygen octahedra. To examine the structural evolution across the interfaces, we performed atomically resolved annular brightfield (ABF) STEM imaging which allows direct observation of the $\mathrm{RuO}_{6} / \mathrm{TiO}_{6}$ octahedral distortions across the whole film. As shown in Fig. 1b, the BTO layers show bulk-like Ti-O polar displacements reflecting their polar nature. Remarkably, the $\mathrm{RuO}_{6}$ octahedron tilts that exist in bulk are totally suppressed in the SRO layers. Instead, polar displacements are observed as induced by the BTO layers. Figure 1c displays the evolution of the layerby-layer $\mathrm{Ru}-\mathrm{O} / \mathrm{Ti}-\mathrm{O}$ polar displacements $\left(\delta_{\mathrm{B}-\mathrm{O}}\right)$ across the whole film. The Ti-O polar displacements near SRO are about $0.2 \pm$ $0.02 \AA$, and the $\mathrm{Ru}-\mathrm{O}$ polar displacements are smaller, with a maximum value of $0.08 \pm 0.04 \AA$. Moreover, convergent beam electron diffraction (CBED) in STEM mode was also performed to study the lattice distortions across the film. The comparison of experimental and simulated CBED (Supplementary Note 2 and Supplementary Fig. 2) indicates that the BTO blocks exhibit polar displacements and the SRO blocks have polar displacements plus octahedron rotations about the $c$-axis, consistent with our observations in Fig. 1. The direction of polarization is opposite in the BTO layers that sandwich the SRO layer, as indicated by the blue arrows in Fig. 1c, a head-to-head polar configuration is observed $^{25}$. From both STEM and second harmonic generation measurements (Supplementary Note 3), we illustrate that such head-to-head polar configuration is the dominant phase in our heterostructure.

In-plane lattice parameters of the bulk BTO and SRO are larger than STO substrate $\left(a_{\mathrm{STO}}=3.905 \AA, a_{\mathrm{SRO}} \sim 3.925 \AA\right.$, and $a_{\mathrm{BTO}}$ $\sim 3.992 \AA)^{26,27}$, indicating that the film is subjected to compressive strain. The in-plane lattice parameters of SRO and BTO films were measured to be $3.90 \pm 0.02 \AA$ (Supplementary Fig. 4), revealing that the film grows coherently on the STO substrate as confirmed by Xray diffraction data (Supplementary Fig. 5). As shown in Fig. 1d, the out-of-plane lattice parameters were measured to be $4.19 \pm$ $0.02 \AA$ for BTO, and $3.95 \pm 0.02 \AA$ for SRO, larger than those of the bulk values $\left(c_{\mathrm{BTO}} \sim 4.036 \AA\right.$, $\mathrm{c}_{\mathrm{SRO}} \sim 3.926 \AA$ ) due to compressive strain. The elongation of out-of-plane lattice parameters should produce an enhanced polarization in BTO and suppress octahedral tilts in $\mathrm{SRO}^{28,29}$. On the other hand, the reduced in-plane lattice parameters of the SRO film favor enhanced octahedral rotation about the $c$-axis ${ }^{28}$. These results are in agreement with the structural feature observed in ABF-STEM images.

Transport and magnetic properties. Bulk SRO is a good metal with low resistivity value in TMO families ${ }^{30}$. In epitaxial thin film, SRO becomes insulating below a critical thickness of 4-5 u.c. ${ }^{31,32}$. Figure 2 a shows resistivity data of the BTO 9/3/ 10 sample as a function of temperature. A metallic behavior is clearly observed with resistivity in the order of $\sim 100 \mu \Omega \mathrm{cm}$ in the measured temperature range. The small upturn in resistivity below $40 \mathrm{~K}$ is likely due to the localization effect of charge carriers as widely observed in ultrathin oxide films ${ }^{33}$. For comparison, a highly insulating behavior is observed in a bare 3-u.c. SRO film grown on a STO substrate. A thick (60 u.c.) SRO film on STO shows a metallic behavior with the resistivity in the order of $10 \mu \Omega \mathrm{cm}$, comparable to bulk value ${ }^{30}$. Remarkably, as shown in Fig. 2a, the BTO $9 / 3 / 10$ has a resistivity that is only within one order of magnitude larger than the bulk value. In contrast, the resistivity of STO 9/3/10 (9-u.c. $\mathrm{SrTiO}_{3} / 3$-u.c. $\mathrm{SrRuO}_{3} / 10$-u.c. $\mathrm{SrTiO}_{3}$ fabricated under the same conditions as BTO 9/3/10) is five times larger than that of BTO $9 / 3 / 10$ at low temperatures and becomes insulating below $90 \mathrm{~K}$. 
a

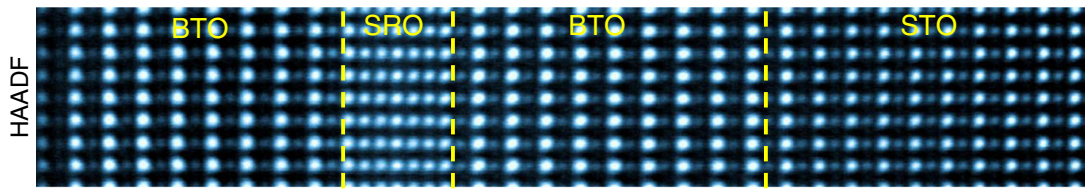

b
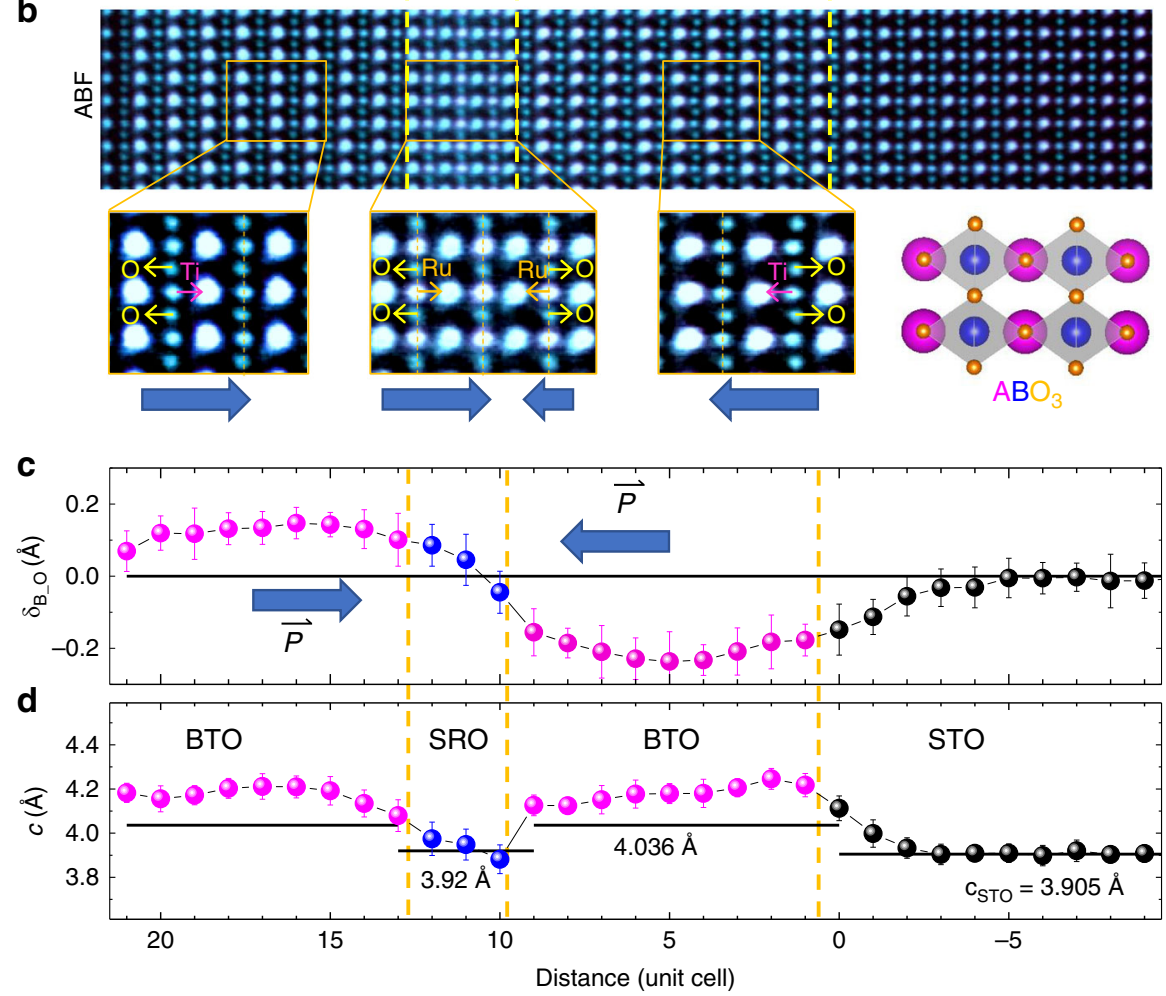

Fig. 1 Direct visualization of interfacial polar distortions in the BTO 9/3/10 film. a HAADF- and $\mathbf{b}$ inversed ABF-STEM images taken along [110] direction. Enlarged ABF-STEM images showing polar distortions in the BTO and SRO blocks. Blue arrows indicate polar displacement direction. $\mathbf{c}$ Variation of polar displacements $\delta_{\mathrm{B}-\mathrm{O}}$ across the whole film. The polar displacements, $\delta_{\mathrm{B}-\mathrm{O}}$, refer to relative displacements between B site cations and $\mathrm{O}$ anions. $\mathbf{d}$ Out-ofplane lattice parameters, $c$, as a function of distance. The measurements were determined by averaging around 60 unit cells parallel to the interface. The error bar shows the standard deviations of the averaged measurements for each vertical atomic layer
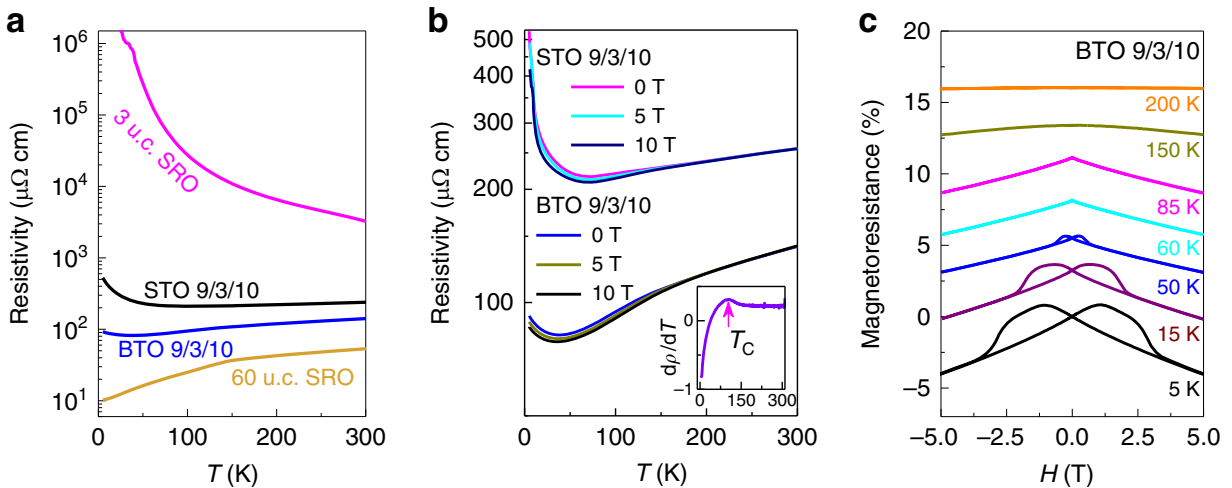

Fig. 2 Transport properties in BTO 9/3/10 and STO 9/3/10. a Temperature dependence of longitudinal resistivity $\rho$ for the BTO 9/3/10, STO 9/3/10, 3 u.c. SRO, and 60 u.c. SRO films. The BTO $9 / 3 / 10$ has low resistivity compared to STO 9/3/10 and bare 3 u.c. SRO film. b Temperature dependence of longitudinal resistivity of BTO $9 / 3 / 10$ and STO $9 / 3 / 10$ under various magnetic field. Metallic behavior is observed at all fields. Inset: temperature dependence of the derivative $\mathrm{d} \rho / \mathrm{d} T$ of BTO 9/3/10. A clear kink around $125 \mathrm{~K}$ is the typical feature of magnetic transition in SRO. c Magnetoresistance of BTO $9 / 3 / 10$ at different temperatures. The butterfly loop indicates ferromagnetism in BTO 9/3/10

We discuss the possible origins on the above observations. As discussed in Supplementary Note 5, both STO 9/3/10 and BTO 9/ $3 / 10$ films have high quality with very low defect densities, ruling out different defect densities as the origin of observed phenomena. We also examined the capping effect by fabricating
3 u.c. SRO on STO substrate capped with 10 u.c. of BTO layers. As shown in Supplementary Fig. 7, the capping effect partially recovers the metallic behavior compared to bare SRO film, it is still more resistive than the BTO $9 / 3 / 10$ samples. Therefore, the observed enhanced conductivity cannot be fully attributed to the 
capping effect. We measured the resistivity of three BTO 9/3/ 10 samples and similar transport behaviors are observed (Supplementary Fig. 8). To better understand the mechanism on the different transport behavior between BTO 9/3/10 and STO 9/3/10, we performed STEM characterization on the STO 9/3/ 10 sample. As shown in Supplementary Fig. 9, both the STO and SRO layers do not show any polar distortions, consistent with the nonpolar nature of STO. The observed conductivity enhancement in BTO 9/3/10 compared to STO 9/3/10 can be understood in the scenario of interface-induced carrier accumulation in the SRO layers by the electric field effects from ferroelectric BTO layers. The electric field from ferroelectric BTO can electrostatically modulate the carrier concentration in neighboring SRO layers, thus causing the conductivity increase in our system. Such electrostatic-induced conductivity enhancement has been observed by Ahn et al. in PZT/SRO system ${ }^{34}$. In Ahn et al.'s work, the enhancement of conductivity was observed when the polarization of PZT is pointing towards the SRO film, which is consistent with our polarization configurations at both $\mathrm{BTO} / \mathrm{SRO}$ interfaces. In contrast, in STO 9/3/10, this effect is absent since no ferroelectric layers exist as shown in Supplementary Fig. 9. Therefore, it is plausible that ferroelectric electrostatic effect plays one of the most important roles in the improved metallicity in BTO 9/3/10.

Figure 2 b shows the resistivity of BTO $9 / 3 / 10$ and STO 9/3/10 under different magnetic fields applied perpendicular to the film surface. Compared to the zero-field case, the resistivity of BTO 9/ $3 / 10$ under field has little difference at high temperatures, but is suppressed below $T_{\mathrm{C}} \sim 125 \mathrm{~K}$ which is defined as the onset of zero-field $\mathrm{d} \rho / \mathrm{dT}$ (inset), indicating that the magnetoresistance (MR) is negative below $T_{C}$. Figure $2 \mathrm{c}$ displays the field dependence of $\mathrm{MR}$ at constant temperatures above and below $T_{\mathrm{C}}$. Note that negative MR depends almost linearly on $H$ below $T_{C}$, and shows a butterfly shape characteristic below $85 \mathrm{~K}$. These features suggest that BTO 9/3/10 has ferromagnetic order below $T_{\mathrm{C}}{ }^{35,36}$.

To confirm the magnetic nature of BTO 9/3/10, we measure the magnetization $(M)$ as a function of temperature $(T)$ and external magnetic field $(H)$. While BTO is diamagnetic, SRO is known to be ferromagnetic in thick films below $\sim 150 \mathrm{~K}$, but becomes nonmagnetic below a critical film thickness of 4 u.c. ${ }^{31,37}$. As shown in Fig. 3a, the magnetic moment of a bare 3-u.c. SRO film grown on a STO substrate is small and has no sign for any magnetic transition. In contrast, $M(T)$ for BTO $9 / 3 / 10$ reveals a sharp rise below $T_{\mathrm{C}} \sim 125 \mathrm{~K}$, indicating a paramagnetic-to-

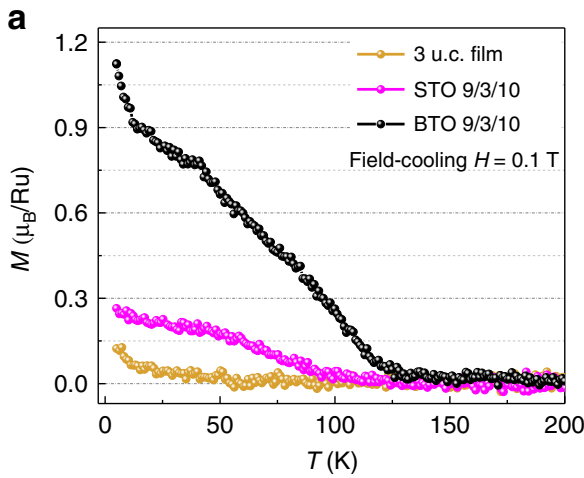

ferromagnetic phase transition. For comparison, the STO 9/3/ 10 shows a magnetic onset around $100 \mathrm{~K}$ with much smaller magnetic moments. To further confirm the ferromagnetic nature of the transition, we examine magnetization hysteresis for BTO 9/ 3/10, STO 9/3/10 and 60 u.c SRO film, as shown in Fig. 3b. At $5 \mathrm{~K}$, the 60 u.c. SRO film shows typical bulk-like ferromagnetic behavior with a saturated magnetic moment of $1.4 \mu_{\mathrm{B}} / \mathrm{Ru}$, corresponding to the low-spin $\mathrm{Ru}$ magnetic state as reported previously $^{30}$. The STO 9/3/10 only shows a small hysteresis loop with saturated magnetic moment of $0.6 \mu_{\mathrm{B}} / \mathrm{Ru}$, confirming its weak ferromagnetism and consistent with a previous publication $^{38}$. Remarkably, the BTO $9 / 3 / 10$ shows ferromagnetic hysteresis loop with a large saturated magnetic moment of $2.3 \mu_{\mathrm{B}} / \mathrm{Ru}$ at $5 \mathrm{~T}$.

Two interesting features can be captured from the magnetization data. First, in BTO $9 / 3 / 10$, the $M(H)$ hysteresis loop is unusual, revealing a steep rise around $2 \mathrm{~T}$ (drop around $-2 \mathrm{~T}$ ) before reaching the saturation value of $2.3 \mu_{\mathrm{B}} / \mathrm{Ru}$. The $M(H)$ highly resembles the hysteresis loop of coupled soft/hard magnetic bilayer system ${ }^{39}$. In our SRO, the soft and hard magnetic components are likely originated from different amplitudes or direction of structure distortions in the middle SRO unit cell and two SRO unit cells near the interfaces (Fig. 1c). When the field is larger than $3 \mathrm{~T}$, the Zeeman energy is large enough to align the spin in different layers, resulting in high saturation moment. Nonetheless, the high saturation moment has not been observed before in ultrathin SRO and is clearly beyond the saturation moment in bulk crystal or thick SRO films ${ }^{30}$. This feature indicates that BTO $9 / 3 / 10$ is in favor of ferromagnetism in making the saturation moment close to the effective $S=1$, which corresponds to the intermediate spin state of $\mathrm{Ru}^{4+}$. We also performed EELS measurements and determined that the valence of $\mathrm{Ti}$ remains close to $4+$, further excluding the magnetic contribution from BTO layers (Supplementary Fig. 10). Second, in the weak ferromagnetic STO 9/3/10 sample, both STO layers are nonpolar so that no polar distortions are induced in the bracketing 3-u.c. SRO layer. Replacing the STO layers with BTO layers successfully induces not only polar distortions in the 3-u.c. SRO, but also strong ferromagnetism with high saturation moment.

\section{Discussion}

Given the experimental observations that we can drive the SRO layer into a ferromagnetic polar metal configuration, it would be helpful to have a first-principles calculation to improve our

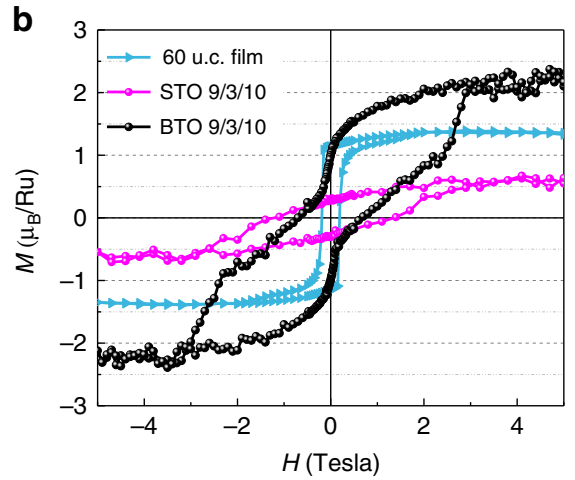

Fig. 3 Unusual magnetism in BTO 9/3/10. a Temperature dependence of magnetization for BTO 9/3/10, STO 9/3/10 and a bare 3 u.c. SRO film under field-cooling (FC) measured by applying a magnetic field $H=0.1 \mathrm{~T}$ normal to the film plane. A pronounced magnetic transition at $125 \mathrm{~K}$ is shown in BTO $9 /$ 3/10. In contrast, STO $9 / 3 / 10$ and bare 3 u.c. SRO show small magnetic moments. b Magnetic hysteresis loop measured at $5 \mathrm{~K}$ for the BTO $9 / 3 / 10$ with saturated moment of $\sim 2.3 \mu_{\mathrm{B}} / \mathrm{Ru}$. The STO 9/3/10 shows weak ferromagnetism with saturated moment of $\sim 0.6 \mu_{\mathrm{B}} / \mathrm{Ru}$ and the 60 u.c. SRO film shows normal ferromagnetism with saturated moment of $\sim 1.4 \mu_{\mathrm{B}} /$ Ru. Note that BTO and STO are diamagnetic in the entire temperature range investigated here 
understanding of the current system. In bulk SRO or thick SRO films, DFT-based calculations are capable of explaining observed properties without using a Hubbard- $U$ parameter or by using a very small $U$ of $0.6 \mathrm{eV}^{40-42}$. In contrast, when the thickness of SRO is reduced to several unit cells, the theoretical calculations are inconclusive. Si et al. illustrated that DFT $+U$ fails to explain the properties of ultrathin SRO film, requiring a DFT + DMFT calculation for better description ${ }^{43}$. Through the calculation, they conclude that ultrathin SRO is an antiferromagnetic insulator and no standard manipulation technique can restore ferromagnetism or metallicity except for carrier doping. Rondinelli et al. also found that $U=0.6 \mathrm{eV}$ works well for bulk SRO, which is a metal, but they were unable to account for the observed metal-toinsulator transition in epitaxial films with thickness smaller than $4-5$ u.c ${ }^{42}$. On the other hand, Gupta et al. pointed out that the metallicity of $3 \mathrm{u}, \mathrm{c}$. SRO film can be retained by subjecting modest compressive strain with a DFT $+U$ calculation with $U=$ $2.5 \mathrm{eV}$ which is a much larger value than bulk $\mathrm{SRO}^{32}$. The authors concluded that this metallic state is highly confined in two dimensions and spin polarized. Similar properties have been predicted using DFT $+U$ calculation by Verissimo-Alves et al. on STO/SRO superlattices with $U=4.0 \mathrm{eV}^{44}$. All the mentioned references showed that octahedral rotations are important in describing the electronic structure.

Given that our thin SRO layer exhibits a unique magnetic polar metal phase which has not been reported before, theoretical explorations regarding the origin of the observed phenomena would be valuable, even in the absence of a proven suitable functional for thin SRO films. Considering the special complexity of our heterostructure including the coupling between different possible distortion modes in SRO, we created a constrained model in an attempt to probe why a 3 u.c. SRO film sandwiched between BTO of opposite ferroelectric polarizations turns into a magnetic polar metal. As described in detail in the Supplementary Note 11 and 12, we have considered initial structures with possible modes guided by the symmetry and optimized the geometry within the LDA $+U$ method. The three structural models in Supplementary Fig. 11b (i, ii, iii) are included in Supplementary Data 1,2, and 3. The end BTO layers are not allowed to relax to ensure that opposite polar displacements are maintained. Finally, we relaxed the remaining structure with different $U$ values. We found that the experimental results can be qualitatively reproduced with $U=3.5 \mathrm{eV}$ (see Supplementary Figs. 11-16).

The approach presented here shows an emerging route to accelerate the discovery towards multifunctional materials with superior properties. Such approach can be easily transferable to a large expansion of other TMO materials such as $\mathrm{Mn}, \mathrm{Cu}, \mathrm{Co}, \mathrm{Ni}$ oxides $^{23}$. It is not only possible to induce a polar phase in ultrathin oxide compounds, but also to manipulate various types of electronic and magnetic interactions. For example, one of the immediate future developments is to switch the relative orientation of two BTO polarizations to control the structural, electronic, and magnetic properties of the bracketing SRO. Such development can open up the possibility to explore a branch of multiferroics by controlling magnetic and electronic properties in a metallic phase ${ }^{19}$. A recent publication shows active control of interfacial skyrmions in bilayer $\mathrm{BTO} / \mathrm{SRO}$, illustrating the importance of such interfaces ${ }^{21}$. We also anticipate this approach to bring further fruitful study to explore functionalities such as magnetoelectric resistive switching memories and ultrathin magnetism manipulation, etc.

In summary, we report a previously unknown magnetic polar metal phase in a complex-oxide heterostructure. The polar distorted SRO shows high conductivity compared to nonpolar SRO of the same thickness. Moreover, a ferromagnetic state with high saturation moment is observed. Polar distortions are found to be responsible for the emergent ferromagnetism, revealing the intrinsic structure-property relationship. The present findings show an emergent route towards designing materials with multiple functionalities.

\section{Methods}

Film growth, x-ray diffraction, and magnetic measurements. The BTO/SRO/ BTO sandwich was grown on $\mathrm{SrTiO}_{3}(001)$ single crystal substrates using stoichiometric targets of $\mathrm{BaTiO}_{3}$ and $\mathrm{SrRuO}_{3}$ by pulsed laser deposition. The substrates were treated for $30 \mathrm{~s}$ in buffered hydrogen fluoride and annealed at $950{ }^{\circ} \mathrm{C}$ in $0.1 \mathrm{MPa}$ oxygen atmosphere. During growth, the substrate temperature was held at $700{ }^{\circ} \mathrm{C}$ with laser energy density $\sim 1 \mathrm{~J} \mathrm{~cm}^{-2}$. BTO was grown at $10 \mathrm{mTorr}$ and SRO was grown at 80 mTorr, both mixed with $1 \%$ ozone. Reflection high-energy electron diffraction (RHEED) was used during growth to monitor oscillations and surface quality. After growth, samples were cooled down to room temperature at 100 mTorr oxygen/o-zone atmosphere. X-ray diffraction measurements have been performed on a laboratory double-axis diffractometer. Magnetic measurements are performed in Quantum Design Magnetic Property Measurement System (MPMS) from 10 to $300 \mathrm{~K}$, by using reciprocating sample option (RSO) with the sensitivity of $5 \times 10^{-9} \mathrm{emu}$.

STEM image and EELS spectrum acquisition. Cross-sectional TEM samples were prepared using focused ion beam (FIB) with $\mathrm{Ga}+$ ions to a thickness around $40 \mathrm{~nm}$ and then further milled using Nanomill with low-energy $\mathrm{Ar}+$ ions to remove the surface damage on the samples. The STEM and EELS results presented in the paper were obtained using the $200 \mathrm{kV}$ JEOL ARM electron microscope at Brookhaven National Library equipped with double aberration correctors, a dualenergy-loss spectrometer and a cold field emission source. The atomic-resolved STEM images were collected with a $21 \mathrm{mrad}$ convergent angle $(30 \mu \mathrm{m}$ condenser aperture) and a collection angle of 67-375 mrad for HAADF and 11-23 mrad for ABF imaging. The STEM imaging conditions were optimized for simultaneous EELS acquisition with a probe size of $0.8 \AA$, a convergence semi-angle of $20 \mathrm{mrad}$, and the collection semi-angle of $88 \mathrm{mrad}$. EELS spectroscopy images were obtained across the whole film with a step size of $0.2 \AA$ and a dwell time of 0.05 s per pixel. The position of each atom column in the HAADF- and ABF-STEM images is measured by the two-dimensioanl Gaussian function. The lattice parameters were extracted from A-site positions, and the polar displacements were calculated by oxygen position with respect to the B-site position. The fine structure of the EELS spectrum (Ti-L edge) was obtained with an energy resolution about $0.7 \mathrm{eV}$ with energy dispersion of $0.25 \mathrm{eV}$. Dural EELS mode was used to calibrate the energy level of the core-loss peaks with the zero-loss peak and to detect possible chemica shifts in the films. Background of the EELS spectra were subtracted using a powerlaw function, and multiple scattering was removed by a Fourier deconvolution method.

SHG measurement. SHG measurements were conducted in reflection geometry with an incident angle of $45^{\circ}$ and fluence of $8 \mathrm{~mJ} / \mathrm{cm}^{2}$. The experimental setup is shown in Fig. S3. After reflection, the fundamental field was filtered and the S- and P-polarized components of the generated second harmonic were individually isolated using a Glan-Taylor polarizer. Optical pulses are provided via commercially purchased Solstice Ace Ti:Sapphire amplifier system from Spectra-Physics, generating pulses at $1.55 \mathrm{eV}$ with a width of $<100 \mathrm{fs}$ at a $1 \mathrm{kHz}$ frequency. The second harmonic was detected using a high-sensitivity spectrometer with a charge-coupled device. For polarization anisotropy measurements, each sample crystalline angle was fixed so that the relative angle between the in-plane crystal axis and the fundamental field was identical. The fundamental field polarization was rotated using a high-accuracy rotation stage.

Density-functional calculations and model. First-principles calculations were carried out using $\mathrm{DFT}^{45}+U$ (d-d static Coulomb interaction $)^{46}$ method with projector augmented wave (PAW) potentials ${ }^{47}$ using the PBE exchange-correlation functional $^{48}$, as implemented in the Vienna ab initio simulation package (VASP) ${ }^{49}$. The total energy and Hellman-Feynman forces were converged to $1 \mu \mathrm{eV}$ and $0.02 \mathrm{eV}^{-1}$, respectively. All calculations were performed with a $450-\mathrm{eV}$ energy cutoff and a $\Gamma$-centered $4 \times 4 \times 2 \mathrm{k}$-point mesh. We have considered

$\sqrt{2} a \times \sqrt{2} b \times 16 c$ supercell of the pseudocubic unit cell. The system contains a total of 160 atoms. Here, fixed the $a$ and $b$ are the in-plane lattiec to the bulk $\mathrm{SrTiO}_{3}$ value $\left(a=3.905 \AA\right.$ ). First, we have considered $<\mathrm{P}+/ \mathrm{SrRuO}_{3} / \mathrm{P}$ - $>$ configuration where the SRO region is kept in the cubic structure (i.e., without any distortion in the SRO block) and BTO in the bulk tetragonal structure (i.e. with the polar distortion). The calculated length of $<\mathrm{P}+/ \mathrm{SrRuO}_{3}$ (no distortion) $/ \mathrm{P}->$ configuration from experimentally determined building block $c$-axis lattice parameters (i.e., unit cell $c$-axis $4.19 \AA$ for BTO, and $3.95 \AA$ for SRO, the $c$-axis has been obtained for $6 / 3 / 7$ structure as 13 (u.c) $\times 4.19+3$ (u.c.) $\times 3.95=66.32 \AA$ ) is found to be $66.32 \AA$. We have optimized the length of simulation cell along the $c$-axis of $<\mathrm{P}+/ \mathrm{SrRuO}_{3}$ (no distortion) $/ \mathrm{P}->$ configuration keeping $a$ and $b$ fixed at STO 
in-plane lattice value $(a=3.905 \AA)$ as shown in Supplementary Fig. 13. The DFT optimized length turns out to be $67.36 \AA$ which is great agreement of $(1.56 \%$ of overestimation) compared to experimental $c$-axis. We have kept the $c$-axis of the supercell to $67.36 \AA$ all other calculations.

\section{Data availability}

The data and code that support the findings of this study are available from the corresponding authors upon reasonable request.

Received: 7 December 2018; Accepted: 24 October 2019;

Published online: 20 November 2019

\section{References}

1. Lezaic, M. Materials science: clockwork at the atomic scale. Nature 533, 38-39 (2016).

2. Balachandran, P. V., Young, J., Lookman, T. \& Rondinelli, J. M. Learning from data to design functional materials without inversion symmetry. Nat. Commun. 8, 14282 (2017).

3. Puggioni, D. \& Rondinelli, J. M. Designing a robustly metallic noncenstrosymmetric ruthenate oxide with large thermopower anisotropy. Nat. Commun. 5, 3432 (2014).

4. Anderson, P. W. \& Blount, E. I. Symmetry considerations on martensitic transformations: 'ferroelectric' metals? Phys. Rev. Lett. 14, 217-219 (1965).

5. Benedek, N. A. \& Birol, T. 'Ferroelectric' metals reexamined: fundamental mechanisms and design considerations for new materials. J. Mater. Chem. C. 4, 4000-4015 (2016).

6. Cao, Y. et al. Artificial two-dimensional polar metal at room temperature. Nat. Commun. 9, 1547 (2018).

7. Kunz, M. \& Brown, I. D. Out-of-center distortions around octahedrally coordinated $\mathrm{d}^{0}$ transition metals. J. Solid State Chem. 115, 395-406 (1995).

8. Kolodiazhnyi, T., Tachibana, M., Kawaji, H., Hwang, J. \& TakayamaMuromachi, E. Persistence of ferroelectricity in $\mathrm{BaTiO}_{3}$ through the insulator-metal transition. Phys. Rev. Lett. 104, 147602 (2010).

9. Fujioka, J. et al. Ferroelectric-like metallic state in electron doped $\mathrm{BaTiO}_{3}$. Sci. Rep. 5, 13207 (2015).

10. Gu, J. et al. Coexistence of polar distortion and metallicity in $\mathrm{PbTi}_{1-x} \mathrm{Nb}_{x} \mathrm{O}_{3}$. Phys. Rev. B 96, 165206 (2017).

11. Shi, Y. et al. A ferroelectric-like structural transition in a metal. Nat. Mater. 12, 1024-1027 (2013).

12. Kim, T. H. et al. Polar metals by geometric design. Nature 533, 68-72 (2016).

13. Dagotto, E., Hotta, T. \& Moreo, A. Colossal magnetoresistant materials: the key role of phase separation. Phys. Rep. 344, 1-153 (2001).

14. Chakhalian, J., Millis, A. J. \& Rondinelli, J. Whither the oxide interface. Nat. Mater. 11, 92-94 (2012).

15. Hwang, H. Y. et al. Emergent phenomena at oxide interfaces. Nat. Mater. 11, 103-113 (2012).

16. Zubko, P., Gariglio, S., Gabay, M., Ghosez, P. \& Triscone, J.-M. Interface physics in complex oxide heterostructures. Annu. Rev. Condens. Matter Phys. 2, 141-165 (2011).

17. Mannhart, J. \& Schlom, D. G. Oxide interfaces-an opportunity for electronics. Science 327, 1607-1611 (2010).

18. Rondinelli, J. M., May, S. J. \& Freeland, J. W. Control of octahedral connectivity in perovskite oxide heterostructures: an emerging route to multifunctional materials discovery. MRS Bull. 37, 261-270 (2012).

19. Fei, Z. et al. Ferroelectric switching of a two-dimensional metal. Nature 560, 336-339 (2018).

20. Tokura, Y., Kawasaki, M. \& Nagaosa, N. Emergent functions of quantum materials. Nat. Phys. 13, 1056 (2017).

21. Wang, L. et al. Ferroelectrically tunable magnetic skyrmions in ultrathin oxide heterostructures. Nat. Mater. 17, 1087-1094 (2018).

22. Mundy, J. A. et al. Atomically engineered ferroic layers yield a roomtemperature magnetoelectric multiferroic. Nature 537, 523-527 (2016).

23. Guo, H. et al. Interface-induced multiferroism by design in complex oxide superlattices. Proc. Natl. Acad. Sci. 114, E5062-E5069 (2017).

24. Rijnders, G., Blank, D. H. A., Choi, J. \& Eom, C.-B. Enhanced surface diffusion through termination conversion during epitaxial $\mathrm{SrRuO}_{3}$ growth. Appl. Phys. Lett. 84, 505-507 (2004).

25. Becher, C. et al. Functional ferroic heterostructures with tunable integral symmetry. Nat. Commun. 5, 4295 (2014).

26. Kwei, G. H., Lawson, A. C., Billinge, S. J. L. \& Cheong, S. W. Structures of the ferroelectric phases of barium titanate. J. Phys. Chem. 97, 2368-2377 (1993).

27. Jones, C. W., Battle, P. D., Lightfoot, P. \& Harrison, W. T. A. The structure of $\mathrm{SrRuO}_{3}$ by time-of-flight neutron powder diffraction. Acta Crystallogr. C 45, 365-367 (1989).
28. Lu, W. et al. The role of octahedral tilting in the structural phase transition and magnetic anisotropy in $\mathrm{SrRuO}_{3}$ thin film. J. Appl. Phys. 113, 63901 (2013).

29. Choi, K. J. et al. Enhancement of ferroelectricity in strained $\mathrm{BaTiO}_{3}$ thin films. Science 306, 1005-1009 (2004).

30. Koster, G. et al. Structure, physical properties, and applications of $\mathrm{SrRuO}_{3}$ thin films. Rev. Mod. Phys. 84, 253-298 (2012).

31. Xia, J., Siemons, W., Koster, G., Beasley, M. R. \& Kapitulnik, A. Critical thickness for itinerant ferromagnetism in ultrathin films of $\mathrm{SrRuO}_{3}$. Phys. Rev. B 79, 140407 (2009).

32. Gupta, K., Mandal, B. \& Mahadevan, P. Strain-induced metal-insulator transition in ultrathin films of $\mathrm{SrRuO}_{3}$. Phys. Rev. B 90, 125109 (2014).

33. Liao, Z. et al. Origin of the metal-insulator transition in ultrathin films of $\mathrm{La}_{2}$ ${ }_{3} \mathrm{Sr}_{1 / 3} \mathrm{MnO}_{3}$. Phys. Rev. B 92, 125123 (2015).

34. Ahn, C. H. et al. Ferroelectric field effect in ultrathin $\mathrm{SrRuO}_{3}$ films. Appl. Phys. Lett. 70, 206-208 (1997).

35. Klein, L. et al. Domain wall resistivity in $\mathrm{SrRuO}_{3}$. Phys. Rev. Lett. 84, 6090-6093 (2000)

36. Xing, L. et al. $\mathrm{Mn}$-induced ferromagnetic semiconducting behavior with linear negative magnetoresistance in $\mathrm{Sr}_{4}\left(\mathrm{Ru}_{1-x} \mathrm{Mn}_{x}\right)_{3} \mathrm{O}_{10}$ single crystals. Sci. Rep. 8, 13330 (2018).

37. Ishigami, K. et al. Thickness-dependent magnetic properties and straininduced orbital magnetic moment in SrRuO thin films. Phys. Rev. B 92, 064402 (2015).

38. Bern, F. et al. Structural, magnetic and electrical properties of SrRuO3films and $\mathrm{SrRuO}_{3} / \mathrm{SrTiO}_{3}$ superlattices. J. Phys. Condens. Matter 25, 496003 (2013).

39. Fullerton, E. E., Jiang, J. S. \& Bader, S. D. Hard/soft magnetic heterostructures: model exchange-spring magnets. J. Magn. Magn. Mater. 200, 392-404 (1999).

40. Etz, C. et al. Indications of weak electronic correlations in $\mathrm{SrRuO}_{3}$ from firstprinciples calculations. Phys. Rev. B 86, 064441 (2012).

41. Ryee, S. \& Han, M. J. Magnetic ground state of $\mathrm{SrRuO}_{3}$ thin film and applicability of standard first-principles approximations to metallic magnetism. Sci. Rep. 7, 4635 (2017).

42. Rondinelli, J. M., Caffrey, N. M., Sanvito, S. \& Spaldin, N. A. Electronic properties of bulk and thin film $\mathrm{SrRuO}_{3}$ : search for the metal-insulator transition. Phys. Rev. B 78, 155107 (2008).

43. Si, L., Zhong, Z., Tomczak, J. M. \& Held, K. Route to room-temperature ferromagnetic ultrathin $\mathrm{SrRuO}_{3}$ films. Phys. Rev. B 92, 041108 (2015).

44. Verissimo-Alves, M., García-Fernández, P., Bilc, D. I., Ghosez, P. \& Junquera, J. Highly confined spin-polarized two-dimensional electron gas in $\mathrm{SrRuO}_{3}$ superlattices. Phys. Rev. Lett. 108, 107003 (2012).

45. Kohn, W. \& Sham, L. J. Self-consistent equations including exchange and correlation effects. Phys. Rev. 140, A1133-A1138 (1965).

46. Lichtenstein, V. I. A., F, A. \& A., I. First-principles calculations of the electronic structure and spectra of strongly correlated systems: the LDA + U method. J. Phys. Condens. Matter 9, 767 (1997).

47. Kresse, G. \& Joubert, D. From ultrasoft pseudopotentials to the projector augmented-wave method. Phys. Rev. B 59, 1758-1775 (1999).

48. Perdew, J. P., Burke, K. \& Ernzerhof, M. Generalized gradient approximation made simple. Phys. Rev. Lett. 77, 3865-3868 (1996).

49. Kresse, G. \& Furthmüller, J. Efficient iterative schemes for $a b$ initio totalenergy calculations using a plane-wave basis set. Phys. Rev. B 54, 11169-11186 (1996).

\section{Acknowledgements}

The authors acknowledge insightful discussion with Y. Cao and S.W. Wu. This work was primarily supported by the US Department of Energy (DOE) under Grant No. DOE DESC0002136. The electron microscopic work done at Brookhaven National Laboratory is sponsored by the US DOE Basic Energy Sciences, Materials Sciences and Engineering Division under Contract DE-AC02-98CH10886. Theory was supported by DOE Grant DE-FG02-09ER46554, computation time was provided on the Titan Supercomputer of the Oak Ridge Leadership Computing Facility (Project MAT136, Engineering multifunctionality in oxide heterostructures). S.G. acknowledges the SRM Supercomputing Facility at the SRM Institute of Science and Technology for providing computing facilities.

\section{Author contributions}

H.G. conceived this study and the experiments. M.M. and H.G. grew the samples and performed electronic and magnetic measurements. Z.W. and Y.Z. performed STEM measurements. A.F. and S.G. performed DFT calculations. M.S. performed x-ray measurements. J.T., E.W.P. and M.S. performed SHG measurements. R.J., Y.Z., S.T.P., J.Z., E.W.P. and H.G. supervised the project. All authors discussed the results and prepared the manuscript.

\section{Competing interests}

The authors declare no competing interests. 


\section{Additional information}

Supplementary information is available for this paper at https://doi.org/10.1038/s41467019-13270-7.

Correspondence and requests for materials should be addressed to S.G., E.W.P. or H.G.

Peer review information Nature Communications thanks Koji Fujita and the other, anonymous, reviewer(s) for their contribution to the peer review of this work.

Reprints and permission information is available at http://www.nature.com/reprints

Publisher's note Springer Nature remains neutral with regard to jurisdictional claims in published maps and institutional affiliations. (c) (i) Open Access This article is licensed under a Creative Commons Attribution 4.0 International License, which permits use, sharing, adaptation, distribution and reproduction in any medium or format, as long as you give appropriate credit to the original author(s) and the source, provide a link to the Creative Commons license, and indicate if changes were made. The images or other third party material in this article are included in the article's Creative Commons license, unles indicated otherwise in a credit line to the material. If material is not included in the article's Creative Commons license and your intended use is not permitted by statutory regulation or exceeds the permitted use, you will need to obtain permission directly from the copyright holder. To view a copy of this license, visit http://creativecommons.org/ licenses/by/4.0/.

(C) The Author(s) 2019 